\title{
Antifungal activity of carvacrol in combination with fluconazole or amphotericin B against Candida albicans
}

\author{
Alireza Khodavandi ${ }^{1}$, Fahimeh Alizadeh ${ }^{2}$, Eskandar Alizadeh ${ }^{2}$ \\ ${ }^{1}$ Department of Biology, Gachsaran Branch, Islamic Azad University, Gachsaran, Iran. \\ ${ }^{2}$ Department of Microbiology, Yasooj Branch, Islamic Azad University, Yasooj, Iran. \\ Email: mnalizadeh@yahoo.com
}

Received 13 August 2017; Received in revised form 25 September 2017; Accepted 27 April 2018

\begin{abstract}
Aims: The aim of this study was to attempt to evaluate the antifungal activity of carvacrol in combination with fluconazole or amphotericin B against Candida albicans.

Methodology and results: Antifungal susceptibilities to carvacrol alone and in combination with fluconazole or amphotericin B were performed using the CLSI standard reference method against clinical isolates of $C$. albicans isolated from the immuno-compromised patients. Proteinase production assay and the expression of genes associated with secreted aspartyl proteinases synthesis (SAP1 and SAP2) were carried out to evaluate the antifungal activity of carvacrol in combination with fluconazole or amphotericin B against $C$. albicans. The carvacrol alone and in combination with fluconazole and amphotericin B exhibited strong inhibitory activity. The carvacrol, exhibited significant synergy and partial synergy with fluconazole or amphotericin B against the test isolates. The data indicated that combination of carvacrol with fluconazole or amphotericin B exerted antifungal effects through reducing SAP enzyme activity. The expression levels of SAP1 and SAP2 genes were down-regulated by carvacrol, fluconazole and amphotericin $\mathrm{B}$ alone. After carvacrol was employed in combination with fluconazole or amphotericin $\mathrm{B}$, the expression levels of $S A P 1$ and $S A P 2$ genes demonstrated the lower expression in comparison to carvacrol alone.

Conclusion, significance and impact of study: These results provide proof of concept for the implementation of carvacrol alone or in combination with fluconazole or amphotericin B inhibitors of $C$. albicans.
\end{abstract}

Keywords: Amphotericin B, Carvacrol, Fluconazole, SAP1, SAP2

\section{INTRODUCTION}

Human fungal infections claim an estimated 1.5 million lives every year. Although the major threats to health are bacterial, viral and parasitic infections, the clinical relevance of fungal infections has not been adequately recognized. Importantly, Candida albicans is a potentially deadly human pathogen in immune-competent and immune-compromised individuals, particularly in patients with hematological malignancies, organ transplant recipients, intensive care unit patients, preterm neonates and patients with various inherited or acquired immune deficiencies (Poulain 2013; Wirnsberger et al., 2016; Kuchler et al., 2016; Lionakis et al., 2017).

The tissue-damaging hydrolytic enzymes such as secreted aspartyl proteinases (SAPs) are considered among the most important virulence factors, which is known to play a central role in the pathogenicity of $C$. albicans. SAP1 to SAP10 genes encode preproenzymes approximately 60 amino acids longer than the mature enzyme in C. albicans (Naglik et al., 2003). Except for
SAP9 and SAP10, which remain bound to the cell wall, SAP 1-8 secreted by $C$. albicans are released into the surrounding medium. SAPs are characterized by broad spectrum proteolytic ability and virulence properties and are differentially expressed at different stages and forms of $C$. albicans growth and disease. Different expression of $S A P$ genes appears to be essential for mucosal and systemic infections. SAPs 1-3 required for adherence of C. albicans to the cell surface and tissue damage, whereas SAP4-SAP6 are associated with systemic disease. Until recently, little is known about $S A P 7$ and $S A P 8$ expression pattern in $C$. albicans pathogenesis. SAP7 and SAP8 were the only genes expressed transiently during $C$. albicans infections. SAP9- and $S A P 10$-encoded proteins maintain cell surface integrity, cell separation and also indirectly contribute to adhesion (Hube and Naglik, 2001; Naglik et al., 2003; Borelli et al., 2007; Cassone, 2013; Staniszewska et al., 2014).

There are currently few antifungals in use include polyenes, azoles, allylamine and echinocandins, among which the oldest class of antifungal drugs is the polyenes 
such as amphotericin B is highly fungicidal and directly bind ergosterol and disrupt the fungal cell membrane. Azoles are the most common agent targeting the synthesis of fungal sterols and, in general, are fungistatic (Prasad et al., 2016).

The ever-increasing rate of resistance of $C$. albicans, poses a serious threat to antifungal therapy, calling for urgent need in search of powerful tool in identifying new antifungal agents that could be used to treat the infections. In the other hand, most of the reported chemicals claimed to possess potential antifungal activates usually had strong potential of resistance reversion in antifungal-resistant $C$. albicans (Shao et al., 2017). Therefore, finding safe natural products to enhance antifungal activity of synthetic antifungal agents can be taken into account as an alternative way to expand the antifungal bank.

Carvacrol $\mathrm{C}_{6} \mathrm{H}_{3} \mathrm{CH}_{3}(\mathrm{OH})\left(\mathrm{C}_{3} \mathrm{H}_{7}\right)$ is a chemical compound of the essential oils of the Lamiaceae family. A monoterpene carvacrol is known to exert potent antimicrobial activity against several microorganisms. Studies have shown that carvacrol has antifungal activity against C. albicans (Vardar-Unlu et al., 2010; Ahmad et al., 2011; Chaillot et al., 2015). The mode of action of the antifungal effect of carvacrol is related to fungal membrane instability by targeting and binding ergosterol (Lima et al., 2013; Chaillot et al., 2015). Some studies have reported the combination of oils with fluconazole or amphotericin B against Candida infections (Khan et al., 2012).

In the present study, we investigated the antifungal activity of carvacrol against clinical isolates of $C$. albicans as well as a standard one with respect to growth inhibition, synergistic effect, proteinase production assay and the expression of genes associated with SAP synthesis in combination with the synthetic antifungal fluconazole or amphotericin B.

\section{MATERIALS AND METHODS}

\section{Anti-microbial agents}

Carvacrol, fluconazole, amphotericin B and chloramphenicol were purchased from Sigma-Aldrich Co. (St. Louis, MO, USA). Dimethyl sulfoxide (100\%) was used to dissolve stock solutions of carvacrol, fluconazole and amphotericin B. These stock solutions were then diluted to the desired concentration based on CLSI M27A3 (Clinical and Laboratory Standards Institute, 2008). The carvacrol and fluconazole or carvacrol and amphotericin $B$ are mixed in $1: 1$ ratio.

\section{Organisms}

Candida albicans ATCC 14053 was provided as a reference quality control. Clinical isolates of $C$. albicans (Ca1-Ca10) were isolated from immuno-compromised patients in Yasooj, Iran. These isolates were preliminarily identified by microscopic and macroscopic morphology, germ tube formation, urease test. Further identification was performed by plated on CHROMagar Candida medium (CHROMagar Company, France). PCR was performed using the universal fungal primers ITS1 (5'TCC GTA GGT GAA CCT GCG G-3') and ITS4 (5'- TCC TCC GCT TAT TGA TAT GC-3'). The identity of the PCR products was confirmed by DNA sequencing method. The sequence similarity was analysed via nucleotide BLAST software (Evans and Richardson, 1989; Harmal et al., 2012). All isolates were stored at $-80^{\circ} \mathrm{C}$ in SDB containing $300 \mu \mathrm{g} / \mathrm{mL}$ of chloramphenicol and sterile $20 \%(\mathrm{v} / \mathrm{v})$ glycerol.

\section{Preparation of inocula}

Preparation of inocula for the broth microdilution testing was performed in accordance with CLSI M27-A3 with RPMI 1640 medium with L-glutamine (Sigma-Aldrich). For each study, after sub-culturing on Sabouraud Dextrose Broth (SDB, Merck Research Laboratories, Darmstadt, Germany). Candida albicans isolate were subcultured onto Sabouraud Dextrose Agar (SDA, Merck Research Laboratories) supplemented with chloramphenicol and passaged at least twice to ensure viability and purity, and incubated at $35{ }^{\circ} \mathrm{C}$ for $24 \mathrm{~h}$. Fungal suspension was prepared based on CLSI M27-A3. Briefly, transferred five colonies more than $1 \mathrm{~mm}$ in diameter to $5 \mathrm{~mL}$ of sterile normal saline and the turbidity of each suspension was adjusted to a $0.5 \mathrm{McF}$ arland standard at $530 \mathrm{~nm}$. The inoculum suspension (a concentration of $1-5 \times 10^{6}$ yeast cells $/ \mathrm{mL}$ ) was diluted to reach a final inoculum of $0.5-5 \times$ $10^{3}$ yeast cells $/ \mathrm{mL}$. For each of the fungal isolates the viability of the yeast was $>97 \%$ by viable pour plate counting method.

\section{Antifungal susceptibility studies}

The minimum inhibitory concentrations (MICs) of carvacrol alone and in combination with fluconazole or amphotericin $B$ were determined with a broth microdilution test according to the reference method of the CLSI (M27A3). The test was carried out using 96-well flat-bottom polystyrene microdilution plates containing $100 \mu \mathrm{L} /$ well of the twofold dilution of the different concentrations of carvacrol (range 6.25-300 $\mathrm{\mu g} / \mathrm{mL}$ ) or fluconazole (range $0.03125-64 \mu \mathrm{g} / \mathrm{mL}$ ) or amphotericin B (range 0.0313-16 $\mu \mathrm{g} / \mathrm{mL}$ ) alone and in combination in RPMI-1640 with Lglutamine (Sigma-Aldrich, St. Louis, MO, USA) buffered to $\mathrm{pH} 7.0$ with $0.165 \mathrm{M}$ morpholinophosphonyl sulfate (MOPS)]. Yeast cells inocula were prepared $(530 \mathrm{~nm}$, abs 0.08-0.1) and diluted to reach a final concentration of 0.5 $5 \times 10^{3}$ yeast cells $/ \mathrm{mL}$ in the wells. Then the plates were incubated at $35^{\circ} \mathrm{C}$ and MICs were measured at $530 \mathrm{~nm}$ from two independent experiments in three separate technical replicates using a Stat Fax 303 Reader (Awareness Technology, Inc., USA) after 24 h. The $\mathrm{MIC}_{90}$ were defined as the lowest concentration of antifungal that caused $90 \%$ growth inhibition compared to control growth.

The fractional inhibitory concentration (FIC) index was equal to (MIC carvacrol in combination/MIC $\mathrm{M}_{\text {carvacrol }}$ 
Malaysian Journal of Microbiology, Vol 14(5) 2018, pp.356-363 DOI: http://dx.doi.org/10.21161/mjm.112217

alone $)+\left(\mathrm{MIC}_{\text {fluconazole }}\right.$ or amphotericin $\mathrm{B}$ in combination/MIC fluconazole or amphotericin B alone), in which synergism was interpreted as FIC $\leq 0.5$, partial synergy was defined when the $\mathrm{FIC}>0.5$ but $<1.0$, additive when $\mathrm{FIC}=1.0$, indifferent when $\mathrm{FIC}>1.0$ but $<4.0$, and antagonistic when FIC $\geq 4.0$ (Khodavandi et al., 2010, 2014).

\section{Proteinase production assay}

Proteinase activity of $C$. albicans treated with carvacrol alone and in combination with fluconazole or amphotericin $B$ were performed at different concentrations based on MIC (2x MIC, $1 \times \mathrm{MIC}, 1 / 2 \times \mathrm{MIC}$ and $1 / 4 \times \mathrm{MIC})$ by the following method: $C$. albicans was grown in $5 \mathrm{~mL}$ YCB + BSA medium (11.7 $\mathrm{g} / \mathrm{L}$ Yeast Carbon Base (Difco Laboratories, Detroit, Michigan, USA), $10 \mathrm{~g} / \mathrm{L}$ glucose; 5 $\mathrm{g} / \mathrm{L}$ bovine serum albumin fraction $\mathrm{V}$ [Sigma-Aldrich]) and placed in a shaker-incubator at $200 \mathrm{rpm}$ and $30{ }^{\circ} \mathrm{C}$ for 72 h. Proteolytic activity was also determined by measuring the increase in trichloroacetic acid soluble products absorbing at $280 \mathrm{~nm}$ in triplicate after $1 \mathrm{~h}$ incubation of the culture supernatant with BSA substrate at $37^{\circ} \mathrm{C}$. The specific activity was expressed as $O_{280} \mathrm{~nm} / \mathrm{OD}_{600} \mathrm{~nm}$ values of the culture. The OD readings equal to or less than 0.02 were considered negative (Macdonald and Odds, 1980).

Table 1: The primers for qRT-PCR.

\begin{tabular}{ccrcc}
\hline Primer & Orientation & Sequence & Length (bp) & Reference \\
\hline SAP1 & Forward & 5' GTTGGTTTTGGTGGTGCTTC 3' & 200 & (Hosseini et al., 2016) \\
& Reverse & 5' GCAGCATTGGGAGAGTTGAG 3' & & (Hosseini et al., 2016) \\
SAP2 & Forward & 5' TGTGGATTCAGGTACCACCA 3' & 192 & (Khodavandi et al., 2011) \\
& Reverse & 5' GCAAATTCGGAAGCTGGA 3' & & (199 \\
& Forward & 5'GAGTTGCTCCAGAAGAACATCCAG 3' & 199 & \\
& Reverse & 5'TGAGTAACACCATCACCAGAATCC 3' & &
\end{tabular}

Table 2: $\mathrm{MIC}_{90}(\mu \mathrm{g} / \mathrm{mL})$ and FIC values of carvacrol alone and in combination with fluconazole or amphotericin B against clinical isolates of Candida albicans.

\begin{tabular}{|c|c|c|c|c|c|c|c|}
\hline \multirow{2}{*}{$\begin{array}{l}\text { Isolates / } \\
\text { Antifungals }\end{array}$} & \multirow{2}{*}{$\frac{\text { Carvacrol }}{\text { MIC }_{90}}$} & \multirow{2}{*}{$\begin{array}{c}\text { Fluconazole } \\
\mathrm{MIC}_{90}\end{array}$} & \multirow{2}{*}{$\frac{\text { Amphotericin B }}{\mathrm{MIC}_{90}}$} & \multicolumn{2}{|c|}{ Carvacrol/ Fluconazole } & \multicolumn{2}{|c|}{ Carvacrol/ Amphotericin B } \\
\hline & & & & $\mathrm{MIC}_{90}$ & $\begin{array}{c}\text { FIC } \\
\text { (Interpretation) }\end{array}$ & $\mathrm{MIC}_{90}$ & $\begin{array}{c}\text { FIC } \\
\text { (Interpretation) }\end{array}$ \\
\hline $\begin{array}{l}\text { C. albicans } \\
\text { ATCC } 14053\end{array}$ & 100 & 16 & 1 & $25 / 2$ & 0.375 (SYN) & $6.25 / 0.125$ & 0.187 (SYN) \\
\hline $\mathrm{Cl}-1$ & 150 & 16 & 1 & $50 / 4$ & 0.583 (PSYN) & $25 / 0.5$ & 0.666 (PSYN) \\
\hline $\mathrm{Cl}-2$ & 100 & 32 & 1 & $50 / 4$ & 0.625 (PSYN) & $6.25 / 0.125$ & 0.187 (SYN) \\
\hline $\mathrm{Cl}-3$ & 150 & 8 & 2 & $50 / 4$ & 0.833 (PSYN) & $25 / 0.5$ & 0.417 (SYN) \\
\hline $\mathrm{Cl}-4$ & 300 & 16 & 2 & $50 / 1$ & 0.229 (SYN) & $25 / 0.5$ & 0.333 (SYN) \\
\hline $\mathrm{Cl}-5$ & 200 & 16 & 4 & $50 / 4$ & 0.50 (PSYN) & $25 / 0.5$ & 0.25 (SYN) \\
\hline $\mathrm{Cl}-6$ & 150 & 16 & 2 & $50 / 4$ & 0.583 (PSYN) & $6.25 / 0.125$ & 0.104 (SYN) \\
\hline $\mathrm{Cl}-7$ & 200 & 32 & 4 & $100 / 8$ & 0.75 (PSYN) & $12.5 / 0.25$ & 0.125 (SYN) \\
\hline $\mathrm{Cl}-8$ & 250 & 32 & 4 & $200 / 16$ & 1.30 (IND) & $25 / 0.5$ & 0.225 (SYN) \\
\hline $\mathrm{Cl}-9$ & 200 & 16 & 1 & $100 / 8$ & 1.00 (ADT) & $12.5 / 0.25$ & 0.312 (SYN) \\
\hline $\mathrm{Cl}-10$ & 300 & 8 & 2 & $100 / 8$ & 1.33 (IND) & $25 / 0.5$ & 0.333 (SYN) \\
\hline
\end{tabular}

$\mathrm{Cl}$ : Clinical isolates of C. albicans; SYN, synergism; PSYN, partial synergism; ADT, additive; IND, indifferent. 
Malaysian Journal of Microbiology, Vol 14(5) 2018, pp.356-363

DOI: http://dx.doi.org/10.21161/mjm.112217

Table 3: Results of proteinase production assay (Means $\mathrm{OD}_{280} \mathrm{~nm} / \mathrm{OD}_{600 \mathrm{~nm}} \pm \mathrm{SD}$ ) of clinical isolates of Candida albicans treated with carvacrol alone and in combination with fluconazole or amphotericin $B$ at different concentration based on MIC.

\begin{tabular}{|c|c|c|c|c|c|c|c|c|c|c|c|}
\hline $\begin{array}{l}\text { Clinical isolates of } C \text {. } \\
\text { albicans/ Concentration } \\
\text { of antifungal agents }\end{array}$ & $\begin{array}{l}\text { C. albicans } \\
\text { ATCC } \\
14053 \\
\end{array}$ & $\mathrm{Cl}-1$ & $\mathrm{Cl}-2$ & $\mathrm{Cl}-3$ & $\mathrm{Cl}-4$ & $\mathrm{Cl}-5$ & $\mathrm{Cl}-6$ & $\mathrm{Cl}-7$ & $\mathrm{Cl}-8$ & $\mathrm{Cl}-9$ & $\mathrm{Cl}-10$ \\
\hline \multicolumn{12}{|l|}{ Carvacrol } \\
\hline $2 \times \mathrm{MIC}$ & $\begin{array}{c}0.03 \pm \\
0.04^{a}\end{array}$ & $\begin{array}{c}0.03 \pm \\
0.08^{\mathrm{a}}\end{array}$ & $\begin{array}{c}0.03 \pm \\
0.01^{\mathrm{a}}\end{array}$ & $\begin{array}{c}0.03 \pm \\
0.09^{a}\end{array}$ & $\begin{array}{c}0.03 \pm \\
0.05^{\mathrm{a}}\end{array}$ & $\begin{array}{c}0.03 \pm \\
0.05^{\mathrm{a}}\end{array}$ & $\begin{array}{c}0.03 \pm \\
0.01^{a}\end{array}$ & $\begin{array}{c}0.03 \pm \\
0.03^{a}\end{array}$ & $\begin{array}{c}0.03 \pm \\
0.09^{a}\end{array}$ & $\begin{array}{c}0.03 \pm \\
0.01^{a}\end{array}$ & $\begin{array}{l}0.03 \pm \\
0.01^{a}\end{array}$ \\
\hline $1 \times \mathrm{MIC}$ & $\begin{array}{l}0.04 \pm \\
0.03^{b}\end{array}$ & $\begin{array}{c}0.03 \pm \\
0.06^{\mathrm{a}}\end{array}$ & $\begin{array}{l}0.04 \pm \\
0.06^{b}\end{array}$ & $\begin{array}{c}0.03 \pm \\
0.05^{\mathrm{a}}\end{array}$ & $\begin{array}{c}0.04 \pm \\
0.02^{b}\end{array}$ & $\begin{array}{c}0.03 \pm \\
0.02^{a}\end{array}$ & $\begin{array}{c}0.03 \pm \\
0.05^{\mathrm{a}}\end{array}$ & $\begin{array}{c}0.04 \pm \\
0.04^{b}\end{array}$ & $\begin{array}{c}0.03 \pm \\
0.01^{\mathrm{a}}\end{array}$ & $\begin{array}{c}0.04 \pm \\
0.06^{\mathrm{b}}\end{array}$ & $\begin{array}{c}0.04 \pm \\
0.01^{b}\end{array}$ \\
\hline $1 / 2 \times M I C$ & $\begin{array}{c}0.05 \pm \\
0.05^{c}\end{array}$ & $\begin{array}{c}0.04 \pm \\
0.06^{b}\end{array}$ & $\begin{array}{c}0.04 \pm \\
0.08^{\mathrm{b}}\end{array}$ & $\begin{array}{c}0.04 \pm \\
0.01^{\mathrm{b}}\end{array}$ & $\begin{array}{c}0.04 \pm \\
0.09^{b}\end{array}$ & $\begin{array}{c}0.04 \pm \\
0.03^{b}\end{array}$ & $\begin{array}{c}0.04 \pm \\
0.07^{b}\end{array}$ & $\begin{array}{c}0.05 \pm \\
0.01^{c}\end{array}$ & $\begin{array}{c}0.04 \pm \\
0.02^{\mathrm{b}}\end{array}$ & $\begin{array}{c}0.05 \pm \\
0.04^{c}\end{array}$ & $\begin{array}{c}0.04 \pm \\
0.01^{b}\end{array}$ \\
\hline $1 / 4 \times \mathrm{MIC}$ & $\begin{array}{c}0.05 \pm \\
0.02^{c}\end{array}$ & $\begin{array}{c}0.05 \pm \\
0.06^{c}\end{array}$ & $\begin{array}{c}0.05 \pm \\
0.06^{c}\end{array}$ & $\begin{array}{c}0.05 \pm \\
0.07^{c}\end{array}$ & $\begin{array}{c}0.05 \pm \\
0.08^{\mathrm{c}}\end{array}$ & $\begin{array}{c}0.05 \pm \\
0.05^{c}\end{array}$ & $\begin{array}{c}0.04 \pm \\
0.05^{\mathrm{b}}\end{array}$ & $\begin{array}{c}0.05 \pm \\
0.03^{c}\end{array}$ & $\begin{array}{c}0.04 \pm \\
0.03^{b}\end{array}$ & $\begin{array}{c}0.05 \pm \\
0.02^{c}\end{array}$ & $\begin{array}{c}0.05 \pm \\
0.01^{c}\end{array}$ \\
\hline $\begin{array}{r}\text { Untreated control } \\
\text { Fluconazole }\end{array}$ & $\begin{array}{l}0.06 \pm \\
0.01^{d}\end{array}$ & $\begin{array}{l}0.06 \pm \\
0.09^{d}\end{array}$ & $\begin{array}{l}0.06 \pm \\
0.02^{d}\end{array}$ & $\begin{array}{l}0.06 \pm \\
0.10^{d}\end{array}$ & $\begin{array}{c}0.05 \pm \\
0.05^{c}\end{array}$ & $\begin{array}{l}0.06 \pm \\
0.03^{d}\end{array}$ & $\begin{array}{c}0.06 \pm \\
0.08^{c}\end{array}$ & $\begin{array}{l}0.06 \pm \\
0.10^{d}\end{array}$ & $\begin{array}{c}0.06 \pm \\
0.04^{\mathrm{c}}\end{array}$ & $\begin{array}{l}0.06 \pm \\
0.07^{d}\end{array}$ & $\begin{array}{l}0.06 \pm \\
0.08^{d}\end{array}$ \\
\hline $2 \times \mathrm{MIC}$ & $\begin{array}{c}0.02 \pm \\
0.01^{\mathrm{a}}\end{array}$ & $\begin{array}{c}0.02 \pm \\
0.06^{a}\end{array}$ & $\begin{array}{c}0.03 \pm \\
0.03^{a}\end{array}$ & $\begin{array}{c}0.02 \pm \\
0.01^{\mathrm{a}}\end{array}$ & $\begin{array}{c}0.02 \pm \\
0.04^{\mathrm{a}}\end{array}$ & $\begin{array}{c}0.02 \pm \\
0.04^{\mathrm{a}}\end{array}$ & $\begin{array}{c}0.02 \pm \\
0.02^{\mathrm{a}}\end{array}$ & $\begin{array}{c}0.02 \pm \\
0.01^{\mathrm{a}}\end{array}$ & $\begin{array}{c}0.02 \pm \\
0.04^{a}\end{array}$ & $\begin{array}{c}0.02 \pm \\
0.05^{\mathrm{a}}\end{array}$ & $\begin{array}{l}0.02 \pm \\
0.03^{a}\end{array}$ \\
\hline $1 \times \mathrm{MIC}$ & $\begin{array}{c}0.03 \pm \\
0.02^{b}\end{array}$ & $\begin{array}{c}0.02 \pm \\
0.05^{\mathrm{a}}\end{array}$ & $\begin{array}{c}0.03 \pm \\
0.01^{\mathrm{a}}\end{array}$ & $\begin{array}{c}0.03 \pm \\
0.06^{\mathrm{b}}\end{array}$ & $\begin{array}{l}0.03 \pm \\
0.01^{\mathrm{b}}\end{array}$ & $\begin{array}{c}0.02 \pm \\
0.07^{a}\end{array}$ & $\begin{array}{c}0.02 \pm \\
0.07^{a}\end{array}$ & $\begin{array}{c}0.03 \pm \\
0.01^{b}\end{array}$ & $\begin{array}{c}0.02 \pm \\
0.04^{\mathrm{a}}\end{array}$ & $\begin{array}{c}0.03 \pm \\
0.07^{b}\end{array}$ & $\begin{array}{c}0.03 \pm \\
0.04^{b}\end{array}$ \\
\hline $1 / 2 \times M I C$ & $\begin{array}{c}0.03 \pm \\
0.09^{b}\end{array}$ & $\begin{array}{c}0.03 \pm \\
0.06^{b}\end{array}$ & $\begin{array}{c}0.04 \pm \\
0.03^{b}\end{array}$ & $\begin{array}{c}0.04 \pm \\
0.04^{c}\end{array}$ & $\begin{array}{c}0.03 \pm \\
0.09^{b}\end{array}$ & $\begin{array}{c}0.03 \pm \\
0.07^{b}\end{array}$ & $\begin{array}{l}0.03 \pm \\
0.04^{\mathrm{b}}\end{array}$ & $\begin{array}{l}0.03 \pm \\
0.01^{b}\end{array}$ & $\begin{array}{l}0.03 \pm \\
0.05^{b}\end{array}$ & $\begin{array}{c}0.03 \pm \\
0.09^{b}\end{array}$ & $\begin{array}{l}0.03 \pm \\
0.06^{b}\end{array}$ \\
\hline $1 / 4 \times \mathrm{MIC}$ & $\begin{array}{c}0.04 \pm \\
0.06^{c}\end{array}$ & $\begin{array}{c}0.04 \pm \\
0.05^{c}\end{array}$ & $\begin{array}{c}0.04 \pm \\
0.05^{\mathrm{b}}\end{array}$ & $\begin{array}{c}0.05 \pm \\
0.02^{d}\end{array}$ & $\begin{array}{c}0.04 \pm \\
0.02^{\mathrm{c}}\end{array}$ & $\begin{array}{c}0.04 \pm \\
0.01^{\mathrm{c}}\end{array}$ & $\begin{array}{c}0.04 \pm \\
0.01^{c}\end{array}$ & $\begin{array}{c}0.04 \pm \\
0.01^{\mathrm{c}}\end{array}$ & $\begin{array}{c}0.05 \pm \\
0.04^{c}\end{array}$ & $\begin{array}{c}0.04 \pm \\
0.09^{c}\end{array}$ & $\begin{array}{c}0.05 \pm \\
0.06^{c}\end{array}$ \\
\hline $\begin{array}{l}\text { Untreated control } \\
\text { Amphotericin B }\end{array}$ & $\begin{array}{l}0.06 \pm \\
0.01^{d}\end{array}$ & $\begin{array}{c}0.06 \pm \\
0.09^{d}\end{array}$ & $\begin{array}{c}0.06 \pm \\
0.02^{c}\end{array}$ & $\begin{array}{c}0.06 \pm \\
0.10^{\mathrm{e}}\end{array}$ & $\begin{array}{l}0.05^{ \pm} \\
0.05^{\mathrm{d}}\end{array}$ & $\begin{array}{l}0.06 \pm \\
0.03^{d}\end{array}$ & $\begin{array}{l}0.06 \pm \\
0.08^{d}\end{array}$ & $\begin{array}{l}0.06 \pm \\
0.10^{\mathrm{d}}\end{array}$ & $\begin{array}{l}0.06 \pm \\
0.04^{\mathrm{d}}\end{array}$ & $\begin{array}{l}0.06 \pm \\
0.07^{d}\end{array}$ & $\begin{array}{l}0.06 \pm \\
0.08^{d}\end{array}$ \\
\hline $2 \times \mathrm{MIC}$ & $\begin{array}{c}0.02 \pm \\
0.01^{a}\end{array}$ & $\begin{array}{c}0.02 \pm \\
0.01^{\mathrm{a}}\end{array}$ & $\begin{array}{c}0.02 \pm \\
0.04^{\mathrm{a}}\end{array}$ & $\begin{array}{c}0.02 \pm \\
0.05^{\mathrm{a}}\end{array}$ & $\begin{array}{c}0.02 \pm \\
0.09^{\mathrm{a}}\end{array}$ & $\begin{array}{c}0.02 \pm \\
0.01^{\mathrm{a}}\end{array}$ & $\begin{array}{c}0.02 \pm \\
0.05^{a}\end{array}$ & $\begin{array}{c}0.02 \pm \\
0.01^{\mathrm{a}}\end{array}$ & $\begin{array}{c}0.02 \pm \\
0.06^{\mathrm{a}}\end{array}$ & $\begin{array}{c}0.02 \pm \\
0.06^{a}\end{array}$ & $\begin{array}{c}0.02 \pm \\
0.08^{\mathrm{a}}\end{array}$ \\
\hline $1 \times \mathrm{MIC}$ & $\begin{array}{c}0.03 \pm \\
0.02^{\mathrm{b}}\end{array}$ & $\begin{array}{c}0.02 \pm \\
0.01^{\mathrm{a}}\end{array}$ & $\begin{array}{l}0.03 \pm \\
0.05^{\mathrm{b}}\end{array}$ & $\begin{array}{c}0.03 \pm \\
0.04^{b}\end{array}$ & $\begin{array}{c}0.02 \pm \\
0.02^{\mathrm{a}}\end{array}$ & $\begin{array}{c}0.03 \pm \\
0.02^{b}\end{array}$ & $\begin{array}{c}0.03 \pm \\
0.05^{\mathrm{b}}\end{array}$ & $\begin{array}{c}0.03 \pm \\
0.01^{b}\end{array}$ & $\begin{array}{c}0.02 \pm \\
0.05^{\mathrm{a}}\end{array}$ & $\begin{array}{c}0.03 \pm \\
0.02^{b}\end{array}$ & $\begin{array}{c}0.03 \pm \\
0.05^{\mathrm{b}}\end{array}$ \\
\hline $1 / 2 \times \mathrm{MIC}$ & $\begin{array}{c}0.04 \pm \\
0.04^{c}\end{array}$ & $\begin{array}{c}0.03 \pm \\
0.01^{\mathrm{b}}\end{array}$ & $\begin{array}{l}0.03 \pm \\
0.04^{\mathrm{b}}\end{array}$ & $\begin{array}{c}0.04 \pm \\
0.04^{\mathrm{c}}\end{array}$ & $\begin{array}{l}0.04 \pm \\
0.02^{\mathrm{b}}\end{array}$ & $\begin{array}{c}0.04 \pm \\
0.01^{\mathrm{c}}\end{array}$ & $\begin{array}{l}0.03 \pm \\
0.06^{b}\end{array}$ & $\begin{array}{c}0.04 \pm \\
0.01^{\mathrm{c}}\end{array}$ & $\begin{array}{l}0.03 \pm \\
0.04^{\mathrm{b}}\end{array}$ & $\begin{array}{c}0.04 \pm \\
0.01^{\mathrm{c}}\end{array}$ & $\begin{array}{l}0.03 \pm \\
0.01^{b}\end{array}$ \\
\hline $1 / 4 \times \mathrm{MIC}$ & $\begin{array}{l}0.05 \pm \\
0.02^{d}\end{array}$ & $\begin{array}{c}0.04 \pm \\
0.03^{c}\end{array}$ & $\begin{array}{c}0.04 \pm \\
0.05^{c}\end{array}$ & $\begin{array}{c}0.04 \pm \\
0.07^{c}\end{array}$ & $\begin{array}{c}0.05 \pm \\
0.02^{\mathrm{c}}\end{array}$ & $\begin{array}{c}0.04 \pm \\
0.06^{c}\end{array}$ & $\begin{array}{c}0.04 \pm \\
0.02^{\mathrm{c}}\end{array}$ & $\begin{array}{c}0.04 \pm \\
0.04^{c}\end{array}$ & $\begin{array}{c}0.04 \pm \\
0.08^{c}\end{array}$ & $\begin{array}{c}0.04 \pm \\
0.03^{\mathrm{c}}\end{array}$ & $\begin{array}{c}0.04 \pm \\
0.02^{c}\end{array}$ \\
\hline Untreated control & $\begin{array}{c}0.06 \pm \\
0.01^{\mathrm{e}}\end{array}$ & $\begin{array}{c}0.06 \pm \\
0.09^{d}\end{array}$ & $\begin{array}{l}0.06 \pm \\
0.02^{d}\end{array}$ & $\begin{array}{c}0.06 \pm \\
0.10^{d}\end{array}$ & $\begin{array}{l}0.05 \pm \\
0.05^{d}\end{array}$ & $\begin{array}{c}0.06 \pm \\
0.03^{d}\end{array}$ & $\begin{array}{c}0.06 \pm \\
0.08^{d}\end{array}$ & $\begin{array}{c}0.06 \pm \\
0.10^{d}\end{array}$ & $\begin{array}{l}0.06 \pm \\
0.04^{d}\end{array}$ & $\begin{array}{c}0.06 \pm \\
0.07^{d}\end{array}$ & $\begin{array}{l}0.06 \pm \\
0.08^{d}\end{array}$ \\
\hline \multicolumn{12}{|l|}{$\begin{array}{l}\text { Carvacrol/ } \\
\text { Fluconazole }\end{array}$} \\
\hline $2 \times \mathrm{MIC}$ & $\begin{array}{c}0.01 \pm \\
0.02^{\mathrm{a}}\end{array}$ & $\begin{array}{c}0.02 \pm \\
0.01^{\mathrm{a}}\end{array}$ & $\begin{array}{c}0.02 \pm \\
0.02^{\mathrm{a}}\end{array}$ & $\begin{array}{c}0.02 \pm \\
0.01^{a}\end{array}$ & $\begin{array}{c}0.02 \pm \\
0.01^{a}\end{array}$ & $\begin{array}{c}0.02 \pm \\
0.02^{\mathrm{a}}\end{array}$ & $\begin{array}{c}0.02 \pm \\
0.02^{a}\end{array}$ & $\begin{array}{c}0.02 \pm \\
0.01^{a}\end{array}$ & $\begin{array}{c}0.02 \pm \\
0.00^{\mathrm{a}}\end{array}$ & $\begin{array}{c}0.01 \pm \\
0.05^{a}\end{array}$ & $\begin{array}{c}0.02 \pm \\
0.01^{\mathrm{a}}\end{array}$ \\
\hline $1 \times \mathrm{MIC}$ & $\begin{array}{l}0.02 \pm \\
0.02^{b}\end{array}$ & $\begin{array}{c}0.02 \pm \\
0.03^{b}\end{array}$ & $\begin{array}{c}0.02 \pm \\
0.02^{\mathrm{a}}\end{array}$ & $\begin{array}{c}0.02 \pm \\
0.01^{a}\end{array}$ & $\begin{array}{c}0.02 \pm \\
0.01^{a}\end{array}$ & $\begin{array}{c}0.02 \pm \\
0.00^{\mathrm{a}}\end{array}$ & $\begin{array}{c}0.02 \pm \\
0.01^{a}\end{array}$ & $\begin{array}{c}0.02 \pm \\
0.01^{a}\end{array}$ & $\begin{array}{c}0.02 \pm \\
0.01^{a}\end{array}$ & $\begin{array}{c}0.02 \pm \\
0.00^{\mathrm{a}}\end{array}$ & $\begin{array}{c}0.02 \pm \\
0.00^{\mathrm{a}}\end{array}$ \\
\hline $1 / 2 \times \mathrm{MIC}$ & $\begin{array}{l}0.02 \pm \\
0.02^{\mathrm{b}}\end{array}$ & $\begin{array}{c}0.03 \pm \\
0.04^{c}\end{array}$ & $\begin{array}{c}0.02 \pm \\
0.03^{\mathrm{a}}\end{array}$ & $\begin{array}{c}0.03 \pm \\
0.03^{b}\end{array}$ & $\begin{array}{c}0.02 \pm \\
0.01^{a}\end{array}$ & $\begin{array}{c}0.02 \pm \\
0.01^{\mathrm{a}}\end{array}$ & $\begin{array}{c}0.02 \pm \\
0.00^{\mathrm{a}}\end{array}$ & $\begin{array}{l}0.03 \pm \\
0.02^{b}\end{array}$ & $\begin{array}{c}0.03 \pm \\
0.05^{\mathrm{b}}\end{array}$ & $\begin{array}{c}0.03 \pm \\
0.04^{b}\end{array}$ & $\begin{array}{c}0.02 \pm \\
0.07^{\mathrm{a}}\end{array}$ \\
\hline $1 / 4 \times \mathrm{MIC}$ & $\begin{array}{l}0.02 \pm \\
0.02^{b}\end{array}$ & $\begin{array}{c}0.03 \pm \\
0.03^{c}\end{array}$ & $\begin{array}{c}0.02 \pm \\
0.01^{a}\end{array}$ & $\begin{array}{c}0.03 \pm \\
0.02^{b}\end{array}$ & $\begin{array}{c}0.03 \pm \\
0.01^{b}\end{array}$ & $\begin{array}{c}0.03 \pm \\
0.02^{b}\end{array}$ & $\begin{array}{l}0.03 \pm \\
0.04^{\mathrm{b}}\end{array}$ & $\begin{array}{l}0.03 \pm \\
0.01^{b}\end{array}$ & $\begin{array}{l}0.03 \pm \\
0.02^{\mathrm{b}}\end{array}$ & $\begin{array}{c}0.03 \pm \\
0.01^{b}\end{array}$ & $\begin{array}{l}0.03 \pm \\
0.00^{\mathrm{b}}\end{array}$ \\
\hline Untreated control & $\begin{array}{c}0.06 \pm \\
0.01^{c}\end{array}$ & $\begin{array}{l}0.06 \pm \\
0.09^{d}\end{array}$ & $\begin{array}{c}0.06 \pm \\
0.02^{\mathrm{b}}\end{array}$ & $\begin{array}{c}0.06 \pm \\
0.10^{c}\end{array}$ & $\begin{array}{c}0.05 \pm \\
0.05^{c}\end{array}$ & $\begin{array}{c}0.06 \pm \\
0.03^{c}\end{array}$ & $\begin{array}{c}0.06 \pm \\
0.08^{c}\end{array}$ & $\begin{array}{c}0.06 \pm \\
0.10^{c}\end{array}$ & $\begin{array}{c}0.06 \pm \\
0.04^{c}\end{array}$ & $\begin{array}{c}0.06 \pm \\
0.07^{c}\end{array}$ & $\begin{array}{c}0.06 \pm \\
0.08^{c}\end{array}$ \\
\hline \multicolumn{12}{|l|}{$\begin{array}{r}\text { Carvacrol/ } \\
\text { Amphotericin B }\end{array}$} \\
\hline $2 \times \mathrm{MIC}$ & $\begin{array}{l}0.01 \pm \\
0.01^{a}\end{array}$ & $\begin{array}{c}0.02 \pm \\
0.02^{\mathrm{a}}\end{array}$ & $\begin{array}{l}0.02 \pm \\
0.01^{a}\end{array}$ & $\begin{array}{c}0.02 \pm \\
0.01^{a}\end{array}$ & $\begin{array}{l}0.01 \pm \\
0.04^{\mathrm{a}}\end{array}$ & $\begin{array}{c}0.02 \pm \\
0.01^{a}\end{array}$ & $\begin{array}{c}0.02 \pm \\
0.01^{a}\end{array}$ & $\begin{array}{c}0.02 \pm \\
0.03^{a}\end{array}$ & $\begin{array}{l}0.02 \pm \\
0.02^{\mathrm{a}}\end{array}$ & $\begin{array}{c}0.01 \pm \\
0.00^{\mathrm{a}}\end{array}$ & $\begin{array}{l}0.02 \pm \\
0.00^{\mathrm{a}}\end{array}$ \\
\hline $1 \times \mathrm{MIC}$ & $\begin{array}{l}0.02 \pm \\
0.02^{\mathrm{a}}\end{array}$ & $\begin{array}{l}0.02 \pm \\
0.02^{\mathrm{a}}\end{array}$ & $\begin{array}{l}0.02 \pm \\
0.02^{\mathrm{a}}\end{array}$ & $\begin{array}{l}0.02 \pm \\
0.03^{\mathrm{a}}\end{array}$ & $\begin{array}{l}0.02 \pm \\
0.01^{\mathrm{b}}\end{array}$ & $\begin{array}{l}0.02 \pm \\
0.01^{\mathrm{a}}\end{array}$ & $\begin{array}{l}0.02 \pm \\
0.01^{\mathrm{a}}\end{array}$ & $\begin{array}{l}0.02 \pm \\
0.01^{\mathrm{a}}\end{array}$ & $\begin{array}{c}0.02 \pm \\
0.02^{\mathrm{a}}\end{array}$ & $\begin{array}{l}0.02 \pm \\
0.00^{b}\end{array}$ & $\begin{array}{c}0.02 \pm \\
0.00^{\mathrm{a}}\end{array}$ \\
\hline $1 / 2 \times \mathrm{MIC}$ & $\begin{array}{l}0.03 \pm \\
0.02^{\mathrm{b}}\end{array}$ & $\begin{array}{c}0.02 \pm \\
0.01^{\mathrm{a}}\end{array}$ & $\begin{array}{l}0.02 \pm \\
0.05^{\mathrm{a}}\end{array}$ & $\begin{array}{l}0.02 \pm \\
0.05^{\mathrm{a}}\end{array}$ & $\begin{array}{l}0.02 \pm \\
0.01^{b}\end{array}$ & $\begin{array}{l}0.03 \pm \\
0.01^{b}\end{array}$ & $\begin{array}{l}0.02 \pm \\
0.01^{a}\end{array}$ & $\begin{array}{l}0.03 \pm \\
0.02^{b}\end{array}$ & $\begin{array}{l}0.02 \pm \\
0.05^{\mathrm{a}}\end{array}$ & $\begin{array}{l}0.02 \pm \\
0.03^{b}\end{array}$ & $\begin{array}{c}0.02 \pm \\
0.00^{\mathrm{a}}\end{array}$ \\
\hline $1 / 4 \times \mathrm{MIC}$ & $\begin{array}{l}0.03 \pm \\
0.02^{\mathrm{b}}\end{array}$ & $\begin{array}{l}0.02 \pm \\
0.01^{\mathrm{a}}\end{array}$ & $\begin{array}{l}0.03 \pm \\
0.03^{\mathrm{b}}\end{array}$ & $\begin{array}{l}0.03 \pm \\
0.01^{b}\end{array}$ & $\begin{array}{l}0.03 \pm \\
0.04^{c}\end{array}$ & $\begin{array}{l}0.03 \pm \\
0.04^{b}\end{array}$ & $\begin{array}{l}0.02 \pm \\
0.01^{\mathrm{a}}\end{array}$ & $\begin{array}{l}0.03 \pm \\
0.03^{b}\end{array}$ & $\begin{array}{l}0.03 \pm \\
0.02^{\mathrm{b}}\end{array}$ & $\begin{array}{l}0.02 \pm \\
0.03^{b}\end{array}$ & $\begin{array}{l}0.03 \pm \\
0.03^{b}\end{array}$ \\
\hline Untreated control & $\begin{array}{l}0.06 \pm \\
0.01^{c}\end{array}$ & $\begin{array}{l}0.06 \pm \\
0.09^{b}\end{array}$ & $\begin{array}{l}0.06 \pm \\
0.02^{\mathrm{c}}\end{array}$ & $\begin{array}{c}0.06 \pm \\
0.10^{c}\end{array}$ & $\begin{array}{l}0.05 \pm \\
0.05^{d}\end{array}$ & $\begin{array}{l}0.06 \pm \\
0.03^{c}\end{array}$ & $\begin{array}{l}0.06 \pm \\
0.08^{\mathrm{b}}\end{array}$ & $\begin{array}{l}0.06 \pm \\
0.10^{c}\end{array}$ & $\begin{array}{l}0.06 \pm \\
0.04^{\mathrm{c}}\end{array}$ & $\begin{array}{c}0.06 \pm \\
0.07^{\mathrm{c}}\end{array}$ & $\begin{array}{l}0.06 \pm \\
0.08^{\mathrm{c}}\end{array}$ \\
\hline
\end{tabular}




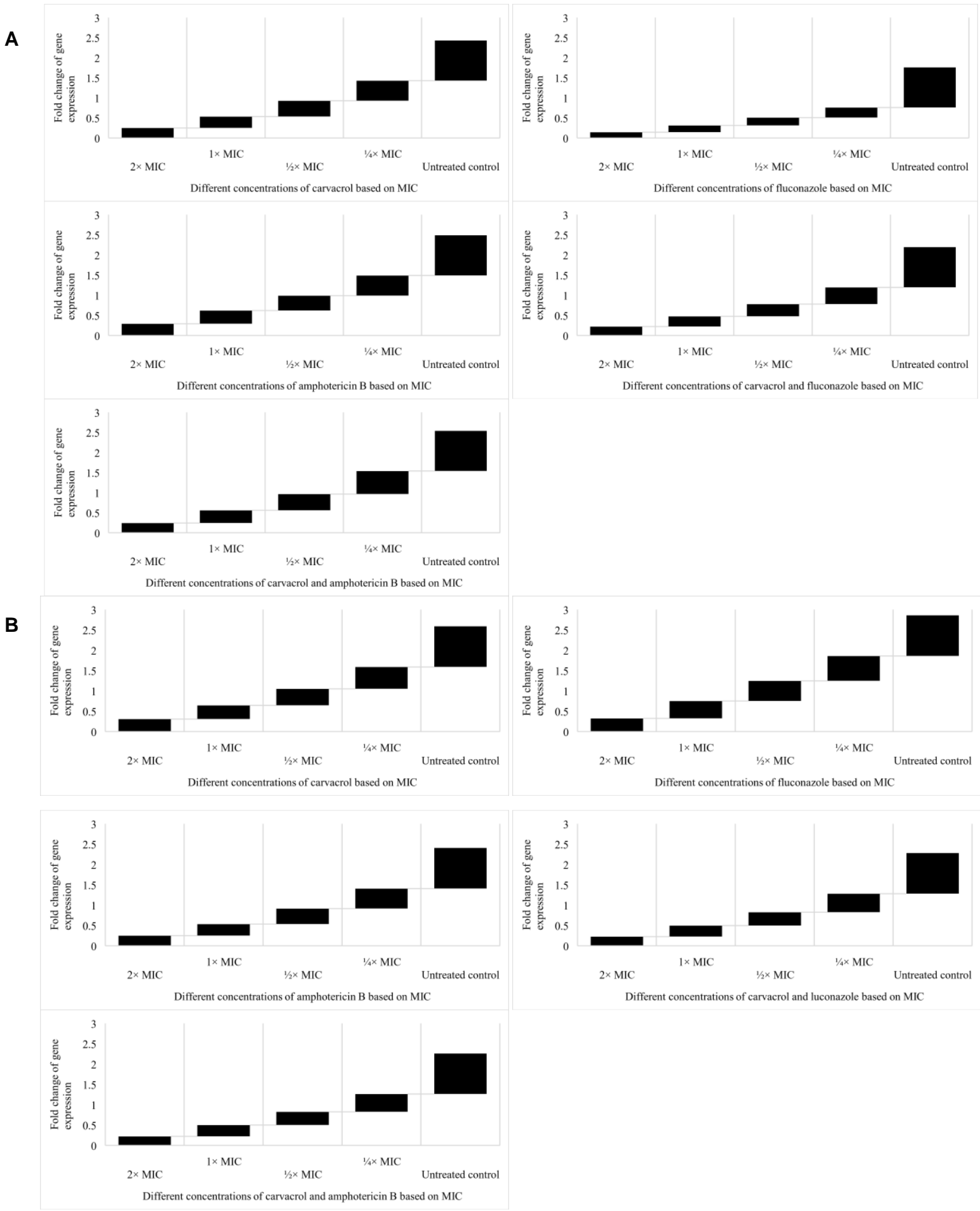

Figure 1: qRT-PCR analysis of SAP1 (A) and SAP2 (B) genes expression in Candida albicans ATCC 14053 treated with carvacrol in combination with fluconazole or amphotericin B at different concentrations based on MIC compared with the untreated control. 
Malaysian Journal of Microbiology, Vol 14(5) 2018, pp.356-363 DOI: http://dx.doi.org/10.21161/mjm.112217

The clinical isolates of $C$. albicans treated with carvacrol alone and in combination with fluconazole or amphotericin B showed decreased proteolytic activity compared to untreated control (Tukey's HSD, $p<0.05$; Table 3). Compared with carvacrol used alone, the proteolytic activity of carvacrol was reduced 1.5-3-fold in concomitant use with fluconazole or amphotericin B.

\section{Impacts of carvacrol alone and in combination with fluconazole or amphotericin B on gene expressions}

In the present study, C. albicans ATCC was treated with different concentrations of carvacrol alone and in combination with fluconazole or amphotericin at different concentrations based on MIC $(2 \times \mathrm{MIC}, 1 \times \mathrm{MIC}, 1 / 2 \times \mathrm{MIC}$ and $1 / 4 \times \mathrm{MIC}$ ). Compared with the untreated control, the expression of SAP1 was down-regulated by $4.00-, 3.52-$, 2.54- and 1.99-fold and SAP2 down-regulated by 3.26-, 2.96-, 2.47- and 1.86-fold after $2 \times$ MIC, $1 \times$ MIC, $1 / 2 \times$ MIC and $1 / 4 \times$ MIC carvacrol treatment, respectively $(p<0.05)$. When $C$. albicans exposed to $2 \times \mathrm{MIC}, 1 \times \mathrm{MIC}, 1 / 2 \times \mathrm{MIC}$ and $1 / 4 \times$ MIC of fluconazole the expression of SAP1 was down-regulated by 6.99-, 5.95-, 5.08- and 3.97-fold and SAP2 down-regulated by 3.06-, 2.35-, 2.01- and 1.64-fold, respectively $(p<0.05)$. The expression level of $S A P 1$ was down-regulated by 3.40-, 3.05-, 2.71- and 1.99-fold and SAP2 down-regulated by 3.97-, 3.55-, 2.63- and 2.03-fold after $2 \times \mathrm{MIC}, 1 \times \mathrm{MIC}, 1 / 2 \times \mathrm{MIC}$ and $1 / 4 \times \mathrm{MIC}$ amphotericin $\mathrm{B}$ treatment, respectively $(p<0.05)$. Under the combined application of $2 \times \mathrm{MIC}, 1 \times \mathrm{MIC}, 1 / 2 \times \mathrm{MIC}$ and $1 / 4 \times \mathrm{MIC}$ of carvacrol and fluconazole, it could be observed that the expression of SAP1 was down-regulated by 4.46-, 3.94-, 3.32- and 2.39-fold and SAP2 down-regulated by 4.41-, $3.69-, 3.05-$ and 2.19 -fold, respectively $(p<0.05)$. The expression of SAP1 was down-regulated by 4.12-, 3.19-, 2.46- and 1.74-fold and SAP2 down-regulated by 4.48-, 3.60-, 3.07- and 2.29-fold, after $2 \times$ MIC, $1 \times$ MIC, $1 / 2 \times$ MIC and $1 / 4 \times$ MIC carvacrol and amphotericin B treatment, respectively $(p<0.05$, Figure 1$)$. In addition, patterns of $C$. albicans gene expression were similar in response to antifungal activity of carvacrol alone and in combination with fluconazole or amphotericin B. Under the combined treatment of carvacrol with fluconazole or amphotericin $\mathrm{B}$, the expression levels of SAP1 and SAP2 genes demonstrated the higher fold change of expression in comparison to carvacrol alone.

\section{DISCUSSION}

Invasive fungal infections have increased in different immuno-compromised populations. Currently, an increase in the number of Candida that are resistant to synthetic antifungal drugs is recognized worldwide; therefore, high throughput screening has emerged as a powerful tool in identifying new antifungal agents that could be used to treat the infections caused by resistant pathogens. Antifungal agents with these characteristics and favourable safety and drug interaction profile would be attractive to evaluate the role of combination therapy for treatment of infections that are difficult to treat (Sardi et al., 2013; Haque et al., 2016; Lionakis et al., 2017). Carvacrol was reported to possess antimicrobial activity against bacteria and also show a certain inhibition on $C$. albicans (Vardar-Unlu et al., 2010; Ahmad et al., 2011; Chaillot et al., 2015).

In this study, we made a rigorous test on the antifungal activity of carvacrol alone and in combination with fluconazole or amphotericin B. Carvacrol alone displayed anti-C. albicans effect compared with fluconazole or amphotericin B. The synergism of carvacrol with fluconazole or amphotericin B against clinical isolates of C. albicans indicated the strong potential of carvacrol to promote the therapy of fluconazole or amphotericin B. Our results are in partial agreement with Doke et al. (2014), who demonstrated that, carvacrol and fluconazole in combination effectively inhibits $C$. albicans biofilm. Khan et al. (2012) demonstrated that, the oils of Cymbopogon martini, i.e., citral and cinnamaldehyde especially eugenol, exhibited significant synergy with fluconazole or amphotericin $\mathrm{B}$, against multi-drug resistant isolates of $C$. albicans. The combination of carvacrol with cinnamaldehyde (Ye et al., 2013), nisin (Periago and Moezelaar, 2001) and ampicillin and nitrofurantoin (Zhang et al., 2011) showed good synergistic antibacterial effect against bacterial strains.

In this study, the proteolytic activity was reduced in $C$. albicans treated with carvacrol in concomitant with fluconazole or amphotericin B. In addition, SAP1 and SAP2 genes associated with SAP synthesis were analyzed by qRT-PCR. As demonstrated, SAP1 and $S A P 2$, both of which were down-regulated in $C$. albicans cells treated with carvacrol alone and in combination with fluconazole or amphotericin B. After carvacrol was employed in combination with fluconazole or amphotericin $\mathrm{B}$, the expression levels of SAP1 and SAP2 genes demonstrated the higher fold change of expression in comparison to carvacrol alone, inferring that the $C$. albicans would produce lower SAPs. Our results are in partial agreement with Hosseini et al., (2016), who demonstrated that, carvacrol is effective in reduction of SAP1-3 expression with a particular effect against fluconazole-resistant $C$. albicans isolates.

In this study, we confirmed that carvacrol could be a candidate of synergist with fluconazole or amphotericin B against clinical isolates of $C$. albicans. Whether these events reflect the potential of carvacrol alone and in combination with fluconazole or amphotericin B for inhibition of SAP synthesis in $C$. albicans which differentially expresses specific gene requires further studies.

\section{ACKNOWLEDGEMENTS}

The authors are thankful to the Islamic Azad University of Yasooj for financial support. Results of the current study are part of an MSc thesis (No. 1195). 
Malaysian Journal of Microbiology, Vol 14(5) 2018, pp.356-363 DOI: http://dx.doi.org/10.21161/mjm.112217

\section{REFERENCES}

Ahmad, A., Khan, A., Akhtar, F., Yousuf, S., Xess, I., Khan, L. A. and Manzoor, N. (2011). Fungicidal activity of thymol and carvacrol by disrupting ergosterol biosynthesis and membrane integrity against Candida. European Journal of Clinical Microbiology and Infectious Diseases 30(1), 41-50.

Borelli, C., Ruge, E., Schaller, M., Monod, M., Korting, H.C., Huber, R. and Maskos, K. (2007). The crystal structure of the secreted aspartic proteinase 3 from Candida albicans and its complex with pepstatin A. Proteins 68(3), 738-748.

Cassone, A. (2013). Development of vaccines for Candida albicans: fighting a skilled transformer. Nature Reviews Microbiology 11(12), 884-891.

Chaillot, J., Tebbji, F., Remmal, A., Boone, C., Brown, G.W., Bellaoui, M. and Sellam, A. (2015). The monoterpene carvacrol generates endoplasmic reticulum stress in the pathogenic fungus Candida albicans. Antimicrobial Agents and Chemotherapy 59(8), 4584-4592.

Clinical and Laboratory Standards Institute. (2008). Reference method for broth dilution antifungal susceptibility testing of yeasts. Approved Standard Document M27-A3, $3^{\text {rd }}$ Edn. CLSI: Wayne, PA: CLSI.

Doke, S. K., Raut, J. S., Dhawale, S. and Karuppayil, S. M. (2014). Sensitization of Candida albicans biofilms to fluconazole by terpenoids of plant origin. The Journal of General and Applied Microbiology 60(5), 163-168.

Evans, E. G. V. and Richardson, M. D. (1989). Medical Mycology: A Practical Approach (The Practical Approach Series). Oxford University Press: New York.

Haque, F., Alfatah, M., Ganesan, K. and Bhattacharyya, M. S. (2016). Inhibitory effect of sophorolipid on Candida albicans biofilm formation and hyphal growth. Scientific Reports 6, 23575.

Harmal, N. S., Khodavandi, A., Alshawsh, M. A., Farida, J., Sekawi, Z., Ng, K.P. and Chong, P. P. (2012). Simplex and triplex polymerase chain reaction (PCR) for identification of three medically important Candida species. African Journal of Biotechnology 11, 12895-12902.

Hosseini, S. S., Yadegari, M. H., Rajabibazl, M. and Ghaemi, E. A. (2016). Inhibitory effects of carvacrol on the expression of secreted aspartyl proteinases 1-3 in fluconazole-resistant Candida albicans isolates. Iranian Journal of Microbiology 8(6), 401-409.

Hube, B. and Naglik, J. (2001). Candida albicans proteinases: resolving the mystery of a gene family. Microbiology 147, 1997-2005. ]

Khan, M.S., Malik, A. and Ahmad, I. (2012). Anticandidal activity of essential oils alone and in combination with amphotericin B or fluconazole against multi-drug resistant isolates of Candida albicans. Medical Mycology 50(1), 33-42.

Khodavandi, A., Alizadeh, F., Aala, F., Sekawi, Z. and Chong, P. P. (2010). In vitro investigation of antifungal activity of allicin alone and in combination with azoles against Candida species. Mycopathologia 169, 287295.

Khodavandi, A., Harmal, N. S., Alizadeh, F., Scully, O. J., Sidik, S. H. M., Othman, F., Sekawi, Z., Ng, K. P. and Chong, P. P. (2011). Comparison between allicin and fluconazole in Candida albicans biofilm inhibition and in suppression of HWP1 gene expression. Phytomedicine 19, 56-63.

Khodavandi, A., Alizadeh, F., Aghai Vanda, N., Karimi, G. and Chong, P. P. (2014). Possible mechanisms of the antifungal activity of fluconazole in combination with terbinafine against Candida albicans. Pharmaceutical Biology 52, 1505-1509.

Kuchler, K., Jenull, S., Shivarathri, R. and Chauhan, N. (2016). Fungal KATs/KDACs: a new highway to better antifungal drugs? PLoS Pathogens 12(11), e1005938.

Lima, I. O., de Oliveira Pereira, F., de Oliveira, W. A., de Oliveira Lima, E., Menezes, E. A., Cunha, F. A. and de Fátima Formiga Melo Diniz, M. (2013). Antifungal activity and mode of action of carvacrol against Candida albicans strains Journal of Essential Oil Research 25, 138-142.

Lionakis, M. S., Iliev, I. D. and Hohl, T. M. (2017). Immunity against fungi. JCI Insight 2(11). pii, 93156.

Macdonald, F. and Odds, F.C. (1980). Inducible proteinase of Candida albicans in diagnostic serology and in the pathogenesis of systemic candidosis. Journal of Medical Microbiology 13, 423-435.

Naglik, J. R., Challacombe, S. J. and Hube, B. (2003). Candida albicans secreted aspartyl proteinases in virulence and pathogenesis. Microbiology and Molecular Biology Reviews 67(3), 400-428.

Periago, P. M. and Moezelaar, R. (2001). Combined effect of nisin and carvacrol at different $\mathrm{pH}$ and temperature levels on the viability of different strains of Bacillus cereus. International Journal of Food Microbiology 68(1-2), 141-148.

Poulain, D. (2013). Candida albicans, plasticity and pathogenesis. Critical Reviews in Microbiology 41, 208-217.

Prasad, R., Shah, A. H. and Rawal, M. K. (2016). Antifungals: mechanism of action and drug resistance. Advances in Experimental Medicine and Biology 892, 327-349.

Sardi, J. C., Scorzoni, L., Bernardi, T., Fusco-Almeida, A. M. and Mendes Giannini, M. J. (2013). Candida species: current epidemiology, pathogenicity, biofilm formation, natural antifungal products and new therapeutic options. Journal of Medical Microbiology 62(Pt 1), 10-24.

Shao, J., Cui, Y., Zhang, M., Wang, T., Wu, D. and Wang, C. (2017). Synergistic in vitro activity of sodium houttuyfonate with fluconazole against clinical Candida albicans strains under planktonic growing conditions. Pharmaceutical Biology 55(1), 355-359.

Staniszewska, M., Bondaryk, M., Malewski, T. and Kurzatkowski, W. (2014). Quantitative expression of Candida albicans aspartyl proteinase genes SAP7, SAP8, SAP9, SAP10 in human serum in vitro. Polish Journal of Microbiology 63(1), 15-20. 
Malaysian Journal of Microbiology, Vol 14(5) 2018, pp.356-363

DOI: http://dx.doi.org/10.21161/mjm.112217

Vardar-Unlu, G., Yağmuroğlu, A. and Unlu, M. (2010). Evaluation of in vitro activity of carvacrol against Candida albicans strains. Natural Product Research 24(12), 1189-1193.

Wirnsberger, G., Zwolanek, F., Asaoka, T., Kozieradzki, I., Tortola, L., Wimmer, R.A., ... Penninger, J.M. (2016). Inhibition of CBLB protects from lethal Candida albicans sepsis. Nature Medicine 22(8), 915-923.

Ye, H., Shen, S., Xu, J., Lin, S., Yuan, Y. and Jones, G.S. (2013). Synergistic interactions of cinnamaldehyde in combination with carvacrol against food-borne bacteria. Food Control 34(2), 619-623.

Zhang, D., Hu, H., Rao, Q. and Zhao, Z. (2011). Synergistic effects and physiological responses of selected bacterial isolates from animal feed to four natural antimicrobials and two antibiotics. Foodborne Pathogens and Disease 8(10), 1055-1062. 